\title{
Humidity Sensing by Polymer-Loaded UHF RFID Antennas
}

\author{
Sabina Manzari, Cecilia Occhiuzzi, Shankar Nawale, Alexandro Catini, \\ Corrado Di Natale, and Gaetano Marrocco
}

\begin{abstract}
Passive ultra high-frequency radio frequency identification tags, besides item labeling, are also able to exploit capability to sense the physical state of the tagged object as well as of the surrounding environment. Here, a new family of polymer-doped tags are proposed and fully characterized for the detection of ambient humidity. A sensitive chemical species based on PEDOT:PSS is used to load a shaped slot, carved into a foldedlike patch tag. The communication and sensing capabilities of the resulting radio-sensor are investigated by means of simulation and measurements that show how to control and balance above opposite requirements by a proper deposition of the sensitive material. The device could have interesting applications in the assessment of the air quality within living and controlled rooms, in the monitoring of the conservation state of foods, in the preservation of walls, and even in the medical field, e.g., to monitor the healing of wounds.
\end{abstract}

Index Terms-Humidity, PEDOT:PSS, polymer, radio frequency identification (RFID), sensor.

\section{INTRODUCTION}

$\mathbf{T}$ HE USE of passive Radio Frequency IDentification (RFID) tags as sensors [1] has been very recently investigated for the wireless observation of several processes in evolution. In particular, sensitive materials may be integrated together with the tag's antenna at the purpose to transduce chemical/physical variation into changes of the tags' radiation performances. Some preliminary experiments with dipoles loaded by carbon nanotubes demonstrated the possibility to sense the presence of toxic gases in the air [2], while the use of shape-memory alloys enabled the detection of temperature thresholds [3]. It is hence feasible to imagine many applications of augmented RFID tags to the observation of the environment if such low-cost passive radio-sensors were pervasively spread within houses, and workplaces in general.

Among different environmental parameters, the observation of humidity is of primarily interest in the assessment of the air quality in living and controlled rooms, in the monitoring of

Manuscript received December 23, 2011; revised March 23, 2012; accepted May 18, 2012. Date of publication June 5, 2012; date of current version August 1, 2012. The associate editor coordinating the review of this paper and approving it for publication was Prof. Kiseon Kim.

S. Manzari, C. Occhiuzzi, and G. Marrocco are with the Department of Computer Science and Systems, University of Rome Tor Vergata, Rome 00133, Italy (e-mail: manzari@disp.uniroma2.it; occhiuzzi@disp.uniroma2.it; marrocco@disp.uniroma2.it).

S. Nawale is with Veermata Jijabat Technology Institute, Mumbai 400019, India (e-mail: shankarnawale125@gmail.com).

A. Catini and C. Di Natale are with the Department of Electronics, University of Rome Tor Vergata, Rome 00133, Italy (e-mail: catini@ing.uniroma2.it; dinatale@uniroma2.it).

Digital Object Identifier 10.1109/JSEN.2012.2202897 the conservation state of foods, in the preservation of walls, exhibition spaces, historical buildings, libraries and archival collections. Additionally, humidity is critical in many biomedical applications concerning the healing degree of wounds [4] and the storage and delivery of pharmaceutical and biological products.

Some early experiments of how integrating humidity sensors into RFID tags have been very recently presented for UHF passive applications. In [5] the sensitive material was simply a blotting paper, eventually doped with $\mathrm{NaCl}$ (salt), covering an RFID patch-like tag. Since the paper absorbs water, the radiation performances of the tag sensibly degrade, thus producing appreciable variations of the tag's response. An inkjet printed tag over a Kapton substrate has been instead experimented in [6]. The sensitivity and the communication performances have been evaluated in terms of turn-on power and frequency shift. These papers demonstrated that the RFID response may be affected by an external chemical receptor reacting to the water absorption. Nevertheless, important issues, concerning the full characterization of the tag as a sensor, are still in question, such as the recovery capabilities in cyclic exposure, the hysteresis, the robustness, the reproducibility, and not last the methodologies to master the sensing performance.

Within this scenario, our work explores for the first time the possibility to integrate polymeric paints into a passive UHF tag with full attention to both communication and sensing issues and their mutual relationships. Polymeric humidity sensors are divided into two fundamental categories according to the dominant sensing mechanisms [7]: resistive-type and capacitivetype. The former reacts to moisture variation by changing its conductivity, while the latter is sensitive to water vapor by varying its dielectric constant [8]. Among the different polymers showing sensitivity to humidity, one of the most exploited is poly (3, 4-ethylenedioxythiophene):poly (styrenesulfonic acid) PEDOT:PSS [9]. Such a conducting polymer shows significant and reversible modification in its electrical properties when exposed to changing humidity. It can be easily manufactured on industrial scale using various deposition techniques [10]. For sensing purposes, it is often deposited into interdigital capacitors and, if characterized in DC, it shows monotonic exponential variation of resistance when exposed to humidity [11]. However, only little knowledge is currently available about the radiofrequency features of the PEDOT:PSS as a sensing material, even without integration with antennas.

In this paper, a tag geometry suitable to host PEDOT:PSS deposition in a controlled way is first described (Section II) together with the basic equations for RFID sensing. 


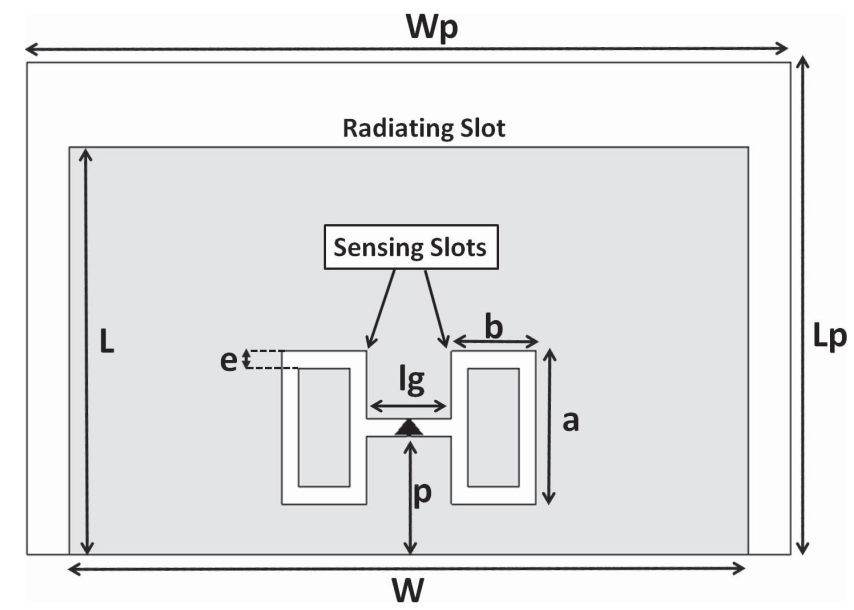

Fig. 1. Layout of the folded patch tag to be used as passive humidity sensor.

The electromagnetic parameters of the polymer are estimated in the UHF RFID band for different grades of humidity exposures (Section III). The achievable sensitivity and the communication performance of the sensor-tag are then numerically and experimentally characterized depending on the amount of deposited polymer (Section IV), and the recovery capability and the hysteresis are quantified in cyclic exposures (Section V). Finally the reliability of the sensortag is preliminarily experimented in a real world application (Section VI).

\section{Humidity SENSOR TAG}

The proposed device is a modified version of the wearable antenna presented in [12]: a folded planar structure over a $4 \mathrm{~mm}$-thick Teflon substrate provided with a radiating edge and a sensing $\mathrm{H}$-shaped slot (Fig. 1). The length $L$ controls the antenna resonances and is about a quarter of wavelength, while the shape factor of the slots can be properly designed to achieve the required input inductive reactance to match the capacitive impedance of the RFID microchip. The humidityreacting polymer will be deposited just inside the slots since they host high value of electric field [2] and are the most sensitive part of the antenna. In order to reduce the area of chemical deposition and further increase the electric field over the apertures, the $\mathrm{H}$-slots are loaded by two rectangular conductive patches. In this way each half glasses-like profile may be considered as a combination of six slot-lines [12] whose characteristic impedances are affected by the dielectric properties of the polymer spread on top (Fig. 2). Since the water absorption produces a change of the polymer's dielectric properties, a variation of both input impedance and losses of the antenna is in turn expected and may be remotely detected by the reader as a modification of the tag's responses.

Finally, the field distribution is not uniform inside the glasses-like slots and hence the most sensitive areas, i.e the portions where the field is maximum, are the central gap (hosting the RFID IC) and the upper horizontal and inner vertical slots.

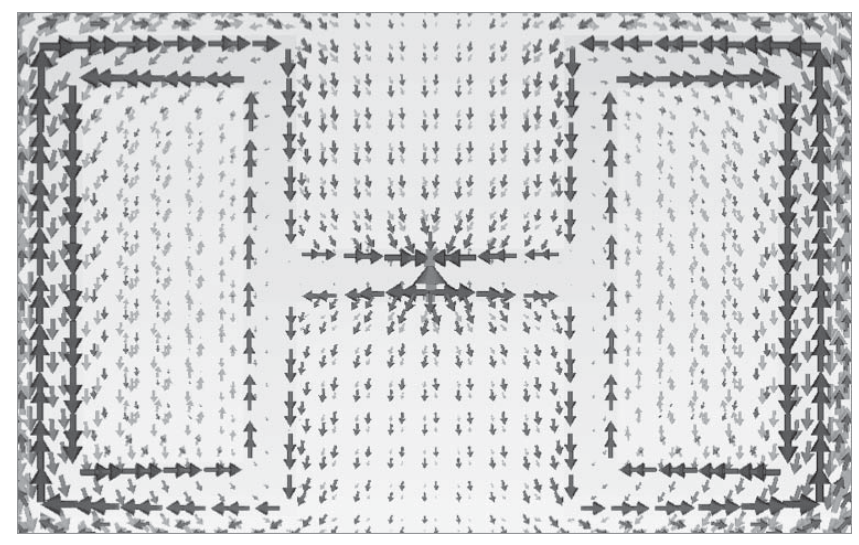

(a)

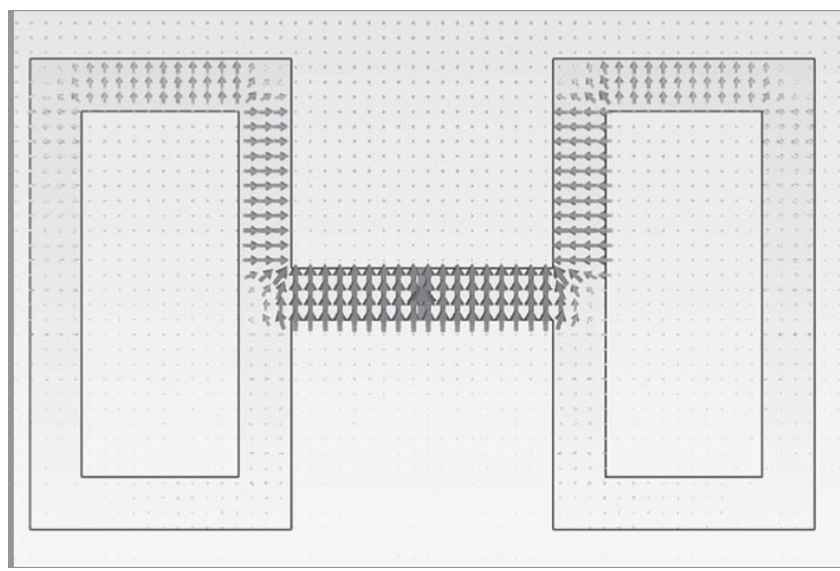

(b)

Fig. 2. (a) Current and (b) aperture field distribution on the sensitive glasses-like slots computed by FDTD.

\section{A. Sensitive Material}

The polymer used is PEDOT:PSS, available commercially as Clevios PH 500, a homogenized water based dispersion [13] generally used for conductive coatings with a PEDOT to PSS ratio of 1:2.5 (Fig. 3). Like other polymers containing sulfonic acid groups, PEDOT:PSS is strongly hygroscopic and takes up moisture when handled under ambient conditions. The polymer dispersion is used to paint the slots; at room temperature, it naturally solidifies so producing a thin film of about $35 \mu \mathrm{m}$ thickness. Since Pedot:PSS is a lossy material, the absorption of electromagnetic energy is higher than in air. Accordingly to this, the electric field reaches higher values when the slots are filled by the polymer with respect to the case they are empty, while maintaining the same trend as in Fig. 2. The polymeric film is able to almost instantaneously absorb water from the environment which is incorporated into the films. Consequently water produces an increase of the layer thickness, especially remarkable for films with high PSS content up to $30 \%$ (as in the considered case).

PEDOT:PSS is well known within the antenna and RF community since, thanks to its characteristics, it has been recently used as conductive material for printed antennas applications [14]. Optical translucency of PEDOT:PSS allows to hide an antenna or make the antenna virtually invisible [15] 

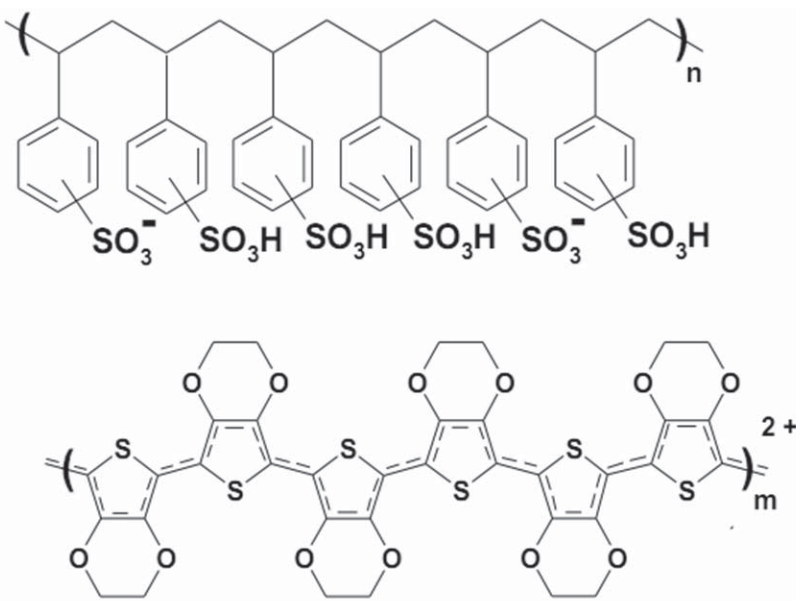

Fig. 3. Poly (3, 4-ethylenedioxythiophene):poly (styrenesulfonic acid) PEDOT:PSS chemical formula.

when mounted on a transparent substrate, such as the screen of a laptop or PDA, while the ductility permits to attach the polymer on flexible fabrics and plastic substrates.

\section{B. Sensing Metrics}

The two-way RFID reader-tag link is strictly dependent on the variation of local relative humidity $R H^{1}$ throughout the change of the PEDOT:PSS features, and accordingly of tag's gain and impedance. It is possible to easily define some sensing indicators carrying information about the humidity change and directly derivable from the reader's measurements. A first parameter is the turn-on power $P_{i n}^{t o}[R H]$, e.g. the minimum input power $P_{i n}$ through the reader's antenna required to activate the tag's integrated circuit (IC)

$$
\begin{aligned}
& P_{i n}^{t o}(\theta, \phi)[R H] \\
& \quad=\left(\frac{4 \pi d}{\lambda_{0}}\right)^{2} \frac{P_{c h i p}}{G_{R}(\theta, \phi) \cdot \eta_{p} \cdot G_{T}(\theta, \phi)[R H] \cdot \tau[R H]}
\end{aligned}
$$

where $d$ is the reader-tag distance, $G_{R}(\theta, \phi)$ is the gain of the reader antenna, $G_{T}(\theta, \phi)[R H]$ is the gain of the tag's antenna, $\eta_{p}$ is the polarization mismatch between the reader and the tag, $P_{\text {chip }}$ is the ICs sensitivity and finally $\tau[R H]$ is the power transmission coefficient of the tag

$$
\tau[R H]=\frac{4 R_{\text {chip }} R_{a}[R H]}{\left|Z_{\text {chip }}+Z_{a}[R H]\right|^{2}}
$$

with $Z_{\text {chip }}=R_{\text {chip }}+j X_{\text {chip }}$ input impedance of the RFID IC and $Z_{A}=R_{a}+j X_{a}$ input impedance of the antenna. From turn-on measurement it is possible to extract the realized gain of the tag $G_{\tau}[R H]$, e.g. the gain of the tag scaled by the mismatch to the IC, strictly correlated to the humidity variation

$$
\begin{aligned}
& G_{\tau}(\theta, \phi)[R H] \\
& =\left(\frac{4 \pi d}{\lambda_{o}}\right)^{2} \frac{P_{c h i p}}{G_{R}\left(\theta_{R}, \phi_{R}\right) \cdot \eta_{p} \cdot P_{i n}^{t o}(\theta, \phi)[R H]} .
\end{aligned}
$$

\footnotetext{
${ }^{1}$ Relative Humidity (RH) is the ratio of the partial pressure of water vapor present in a gas to the saturation vapor pressure of the gas at a given temperature. It is a function of temperature, and thus it is a relative measurement.
}

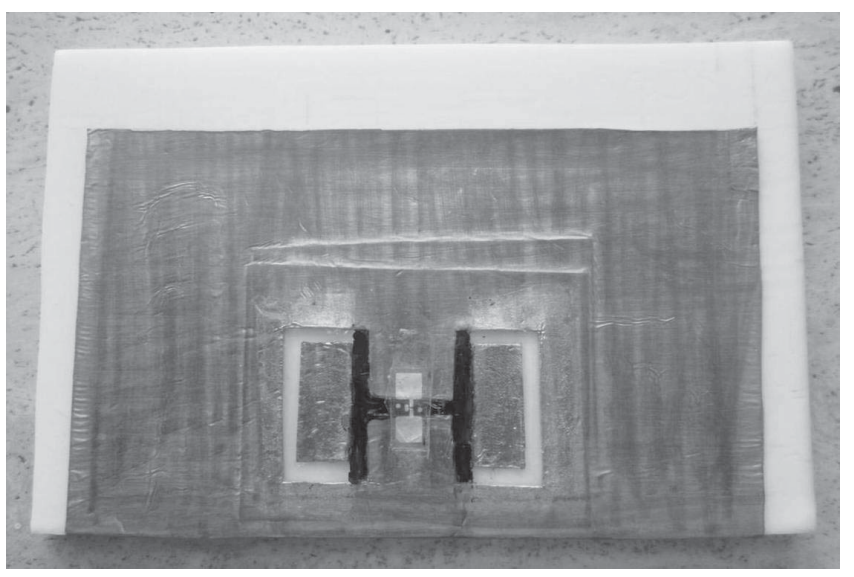

Fig. 4. RFID sensor prototype over a Teflon substrate 4-mm thick with a partial polymer filling (in black) on the sensing glasses-like slots.

TABLE I

Size in Millimeters of the Parameters in Fig. 4.

\begin{tabular}{|c||c|}
\hline Parameter & Value [mm] \\
\hline \hline $\mathrm{a}$ & 18 \\
\hline $\mathrm{b}$ & 10 \\
\hline $\mathrm{e}$ & 2 \\
\hline $\mathrm{p}$ & 14 \\
\hline $\mathrm{L}$ & 48 \\
\hline $\mathrm{Lp}$ & 58 \\
\hline $\mathrm{lg}$ & 10 \\
\hline $\mathrm{W}$ & 80 \\
\hline $\mathrm{Wp}$ & 90 \\
\hline
\end{tabular}

By considering the backward link, it is possible to introduce another sensing metric directly measurable by the reader in terms of the Received Signal Strength Indicator (RSSI): the normalized backscattered power $p_{B S}[R H]$, e.g the ratio between the backscattered power $P_{R \leftarrow T}[R H]$ collected by the reader and the input power $P_{i n}[R H]$ to the reader's antenna

$$
p_{B S}(\theta, \phi)[R H]=\frac{P_{R \leftarrow T}(\theta, \phi)[R H]}{P_{i n}(\theta, \phi)[R H]} .
$$

\section{PRototype AND Characterization}

A first prototype of the polymer-doped tag (Fig. 4) has been designed and fabricated according to the guidelines described in [12] (size listed in Table I). The tag is matched to the G2iL NXP IC with $Z_{c h i p}=25-j 23 \Omega$ and power sensitivity $P_{\text {chip }}=-18 \mathrm{dBm}$. The design procedure has been optimized for the unloaded tag, i.e. without polymer deposition on the glasses-like slots.

The communication performances of the unloaded tag have been characterized for what concerns the realized gain $G_{\tau}$ (3) by means of both simulations and measurements [17]. Simulations have been performed by the help of a Finite-Difference Time-Domain (FDTD) tool, while the measurements have been carried out by means of a UHF long-range reader based on the ThingMagic M5-e ASIC whose output power can be controlled by $0.5 \mathrm{dBm}$ steps. The reader's antenna was a $5 \mathrm{~dB}$ linear polarized patch, placed $50 \mathrm{~cm}$ apart from the radio-sensor. Reflections from ground and side walls were minimized by using absorbing panels. Results are shown in Fig. 5. From (1) it is possible to determine the maximum 


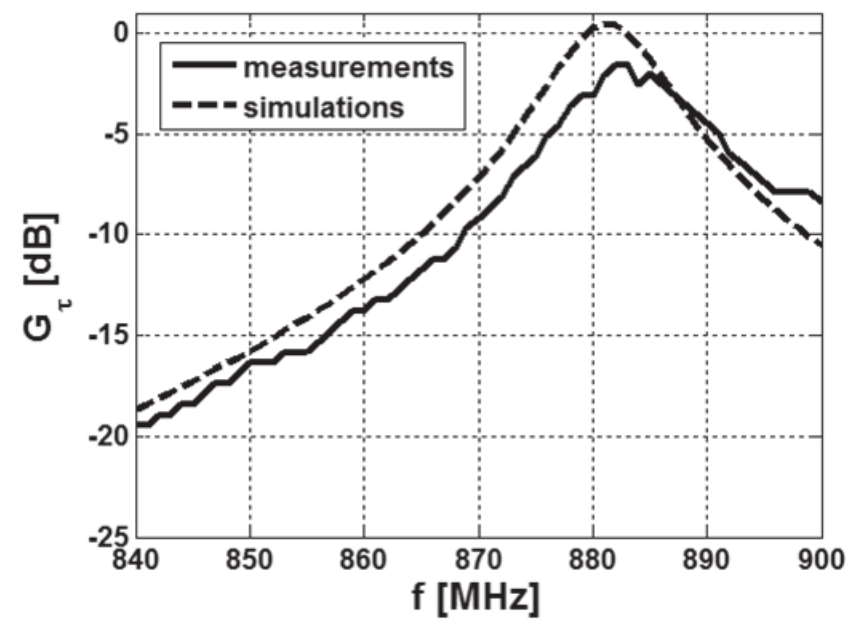

Fig. 5. Measured and simulated realized gain along the antenna axis (broadside observation) for the unloaded sensor tag.

activation distance, $d_{\max }=8 m$, by considering $3.2 \mathrm{~W}$ EIRP, which is the maximum emitted power from the reader allowed by European regulations.

\section{A. Electromagnetic Characterization of PEDOT:PSS}

While there are some electrical information about the PEDOT:PSS in DC [13], no significant data are currently available in the UHF RFID band, thus a specific characterization is required. Furthermore, like many other chemical species [2], the dielectric properties of PEDOT:PSS are strictly dependent on the concentrations, the layer thickness and the deposition techniques and hence the electromagnetic characterization presented here is specific for the considered prototypes. The same methodology may be however applied for other concentration of polymer and deposition modalities.

In the presented analysis the PEDOT:PSS is assumed as a lossy conductor [14] and its unknown conductivity $\sigma_{P}$ is here identified according to the following procedure. The bare tag is doped by the polymer as shown in Fig. 4 and its realized gain $G_{\tau, f_{i}}^{\text {meas }}\left[R H_{n}\right]$ is measured at three frequencies $f_{i}=\{860,870,880\} \mathrm{M} \mathrm{Hz}$ in two different humidity conditions: ambient air $\left(\mathrm{RH}_{1}=50 \%\right)$ and wet air $\left(\mathrm{RH}_{2}=\right.$ $100 \%)$. The second condition has been obtained by placing the tag inside a closed plastic chamber partially filled with water (Fig. 6). When the chamber is closed, the relative humidity approaches $\mathrm{RH}=100 \%$ (wet air), while in case the cover is removed, the humidity is that of the ambient air $(\mathrm{RH}=50 \%)$. The measurements have been performed at room temperature of $24{ }^{\circ} \mathrm{C}$, to be considered stable all along the process. Then, a numerical model of the tag is arranged by means of an FDTD solver able to calculate the realized gain, say $G_{\tau, f_{i}}^{s i m}\left(\sigma_{P}\left[R H_{n}\right]\right)$. The conductivity of the material simulating the PEDOT:PSS deposition is therefore optimized in order to minimize, separately for the two humidity grades, the following error function $\Delta_{t o t}\left[R H_{n}\right]$ :

$$
\begin{aligned}
\sigma_{P, n} & : \Delta_{\text {tot }}\left[R H_{n}\right] \\
& =\sum_{i=1}^{3} \frac{\left|G_{\tau, f_{i}}^{\text {meas }}\left[R H_{n}\right]-G_{\tau, f_{i}}^{\text {sim }}\left(\sigma_{P}\left[R H_{n}\right]\right)\right|^{2}}{\left|G_{\tau, f_{i}}^{\text {meas }}\left[R H_{n}\right]\right|^{2}} \rightarrow 0 .
\end{aligned}
$$

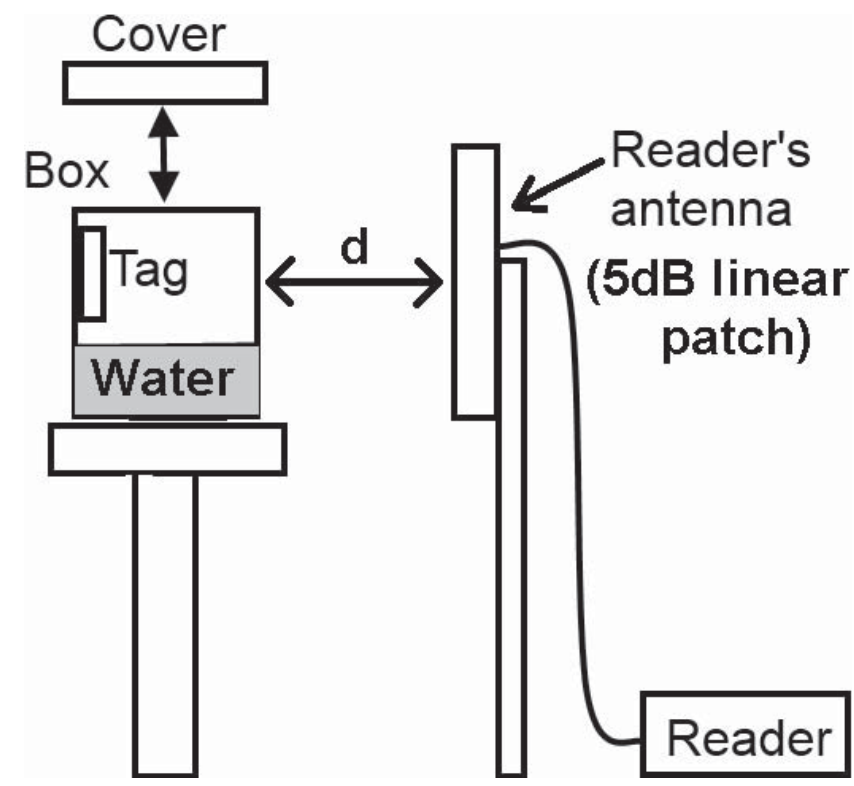

Fig. 6. Measurement setup: the sensor tag is placed into a sealed plastic chamber partially filled with water at a distance $d=50 \mathrm{~cm}$ away from the reader's antenna. The plastic chamber, thanks to its low permittivity, does not significantly affect the response of the tag in either low or high humidity conditions, as verified by comparing simulation and measurement results.

The resulting error profiles for two humidity conditions $(\mathrm{RH}=50 \%$ and $\mathrm{RH}=100 \%)$ are shown in Fig. 7(a). The estimated values of $\sigma_{P}$ minimizing the global error $\Delta_{t o t}$ are hence $\sigma_{P}\left(R H_{1}=50 \%\right)=5 \mathrm{~S} / \mathrm{m}$ and $\sigma_{P}\left(R H_{2}=100 \%\right)=10 \mathrm{~S} / \mathrm{m}$ that means the conductivity of PEDOT:PSS approximately doubles on moving from ambient air to a completely saturated air, sensibly increasing the antenna's losses. To check the validity of the estimated conductivity $\sigma_{P}$, the measured and simulated results for the prototype of Fig. 4 are compared in Fig. 7(b) and a satisfactory agreement is apparent at least around the reference frequency $\mathrm{f}=870 \mathrm{MHz}$. A variation of about $6 \mathrm{~dB}$ and a frequency shift of about $10 \mathrm{MHz}$ is expected during a complete exposure cycle $(R H=50 \rightarrow 100 \%)$.

\section{Communication and Sensing}

Since there is no decoupling from the operative and structural point of view between antenna and sensor, the proposed device has to be characterized and optimized from both perspectives. In particular, the sensing capabilities of a tagas-a-sensor are paid in terms of a worsening of the tag's realized gain (and hence of the read distance) along with the humidity exposure (as shown in Fig. 7). It is hence important to balance two opposite requirements, e.g. to pursue a useful sensitivity at the minimum degradation of the communication. The amount of deposited polymer is expected to be an effective parameter to tune the communication/sensing performances as demonstrated in the following experiments.

\section{A. Communication Performances}

The communication performances of the doped tag have been evaluated at ambient humidity in terms of realized gain $G_{\tau}$ versus the amount of polymer deposition inside the glasses-like slots according to the layouts in Fig. 8. From the 


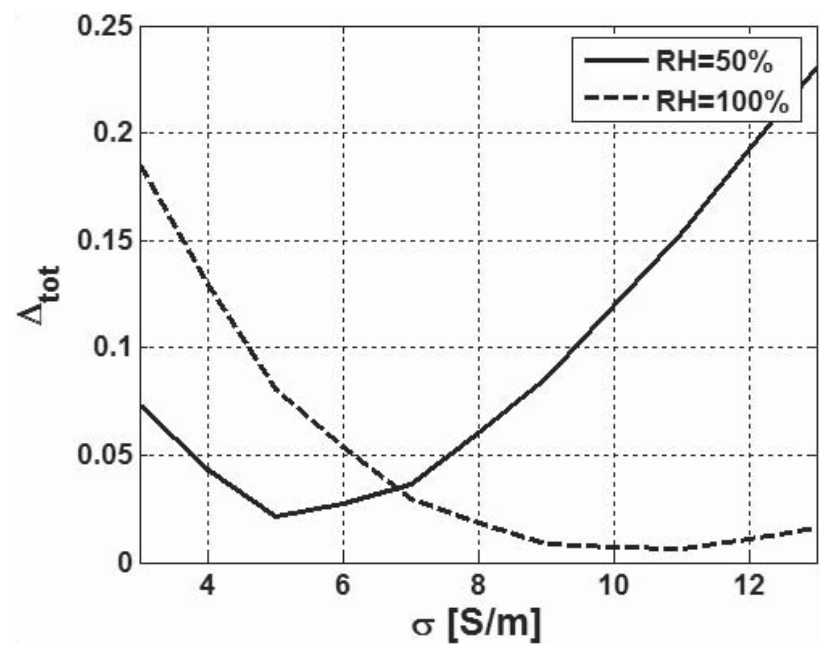

(a)

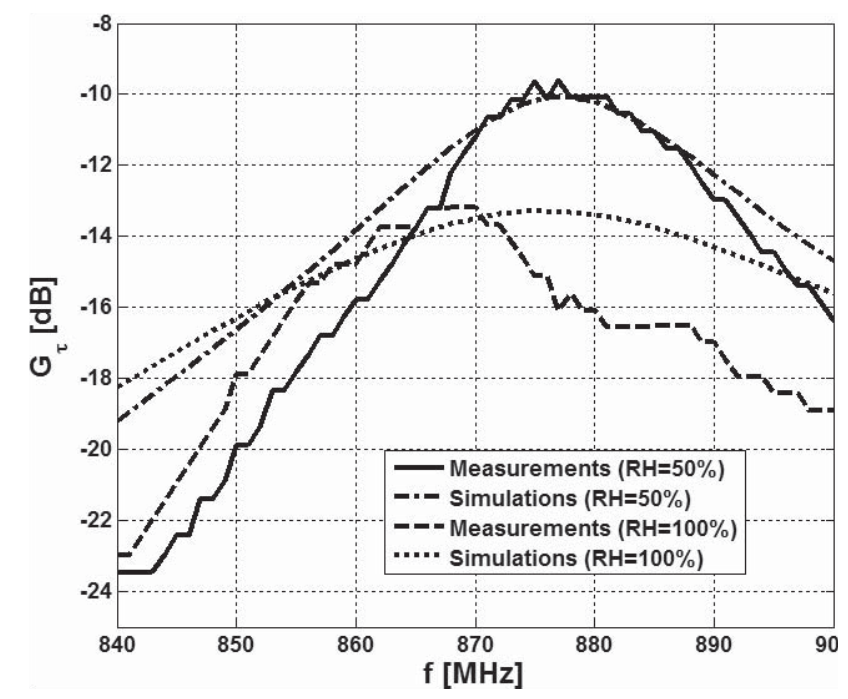

(b)

Fig. 7. (a) Global error in the parametric identification of the PEDOT:PSS conductivity $\sigma_{P}$ for ambient air $(\mathrm{RH}=50 \%)$ and wet air $(\mathrm{RH}=100 \%)$. (b) Measured and simulated $G_{\tau}$ for two different RH levels along the antenna axis (broadside observation) using the identified conductivities.

measured profiles in Fig. 9, it is worth observing that there would be only a negligible degradation in communications on moving from the blank tag to the deposition $\mathrm{F}$ obtained with a single polymer drop just behind the microchip. For the other cases, it is instead apparent that, by filling the central slot and the two upper horizontal slots (as suggested by Fig. 2), the peak of the realized gain sensibly shifts and attenuates of about $8 \mathrm{~dB}$. Accordingly, the activation distance would be reduced from $8 \mathrm{~m}$ to $3.5 \mathrm{~m}$.

\section{B. Sensing Performances}

The sensing performances of the RFID tag have been analyzed dynamically when the humidity inside the box of Fig. 6 gradually changes from ambient conditions up to saturation, for the most representative polymer deposition layouts (cases A, D and F in Fig. 8). The sensors were not conditioned prior to operation. Fig. 10 shows the measured variation of the turn-on power $P_{i n}^{t o}[R H]$ with respect to the increase of

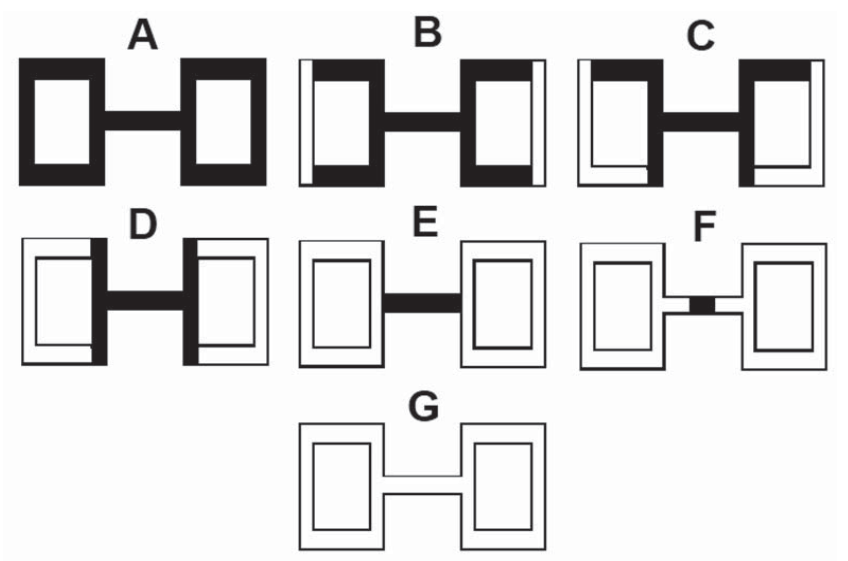

Fig. 8. Some layouts of polymer deposition inside the glasses-like slots. The first geometry (A) corresponds to a complete loading of the slot, the last one $(\mathrm{G})$ to an unloaded (blank) tag.

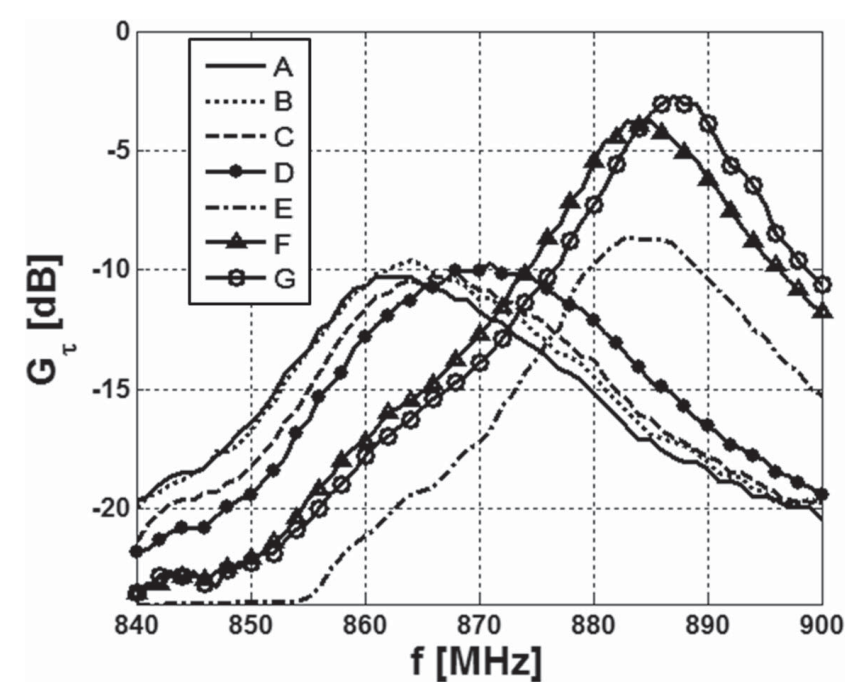

Fig. 9. Measured realized gain along the antenna axis (broadside observation) for different polymer depositions as in Fig. 8.

humidity at the frequencies where, case by case, the turn-on power is minimum. To improve the readability, a mean-square interpolation is superimposed to the stair-step distribution of the measurement data caused by the coarse output power resolution $(0.5 \mathrm{dBm})$ of the UHF reader.

All the measured tags detect the variation of the humidity according to an exponential profile so that the turn-on power increases of about $3.5 d B \div 6 d B$ depending on the specific PEDOT:PSS deposition. The wider is the area covered by the polymer, the greater is the water absorption and thus the dynamic range of the sensor. It is moreover worth noticing that during the very early grade of the exposure, e.g. for $50 \%<\mathrm{RH}<75 \%$ ( $t<10 \mathrm{~min}$ ), the responses of layout $\mathrm{A}$ (full deposition) and layout D (H-like deposition) are indistinguishable while the overall saturation threshold is instead $2 \mathrm{~dB}$ higher for the case A. Finally, even the single-drop layout F offers the possibility to track the humidity change with an interesting overall $3.5 \mathrm{~dB}$ dynamic range.

As for conventional sensors, also in this case it is possible to determine the calibration curves, e.g. the plots of the sensor 
(a)

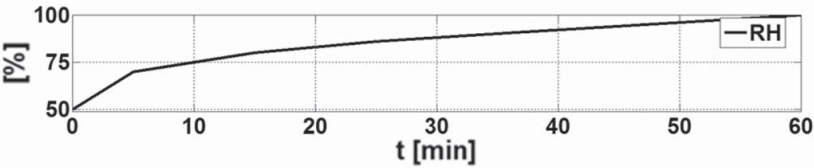

(b)

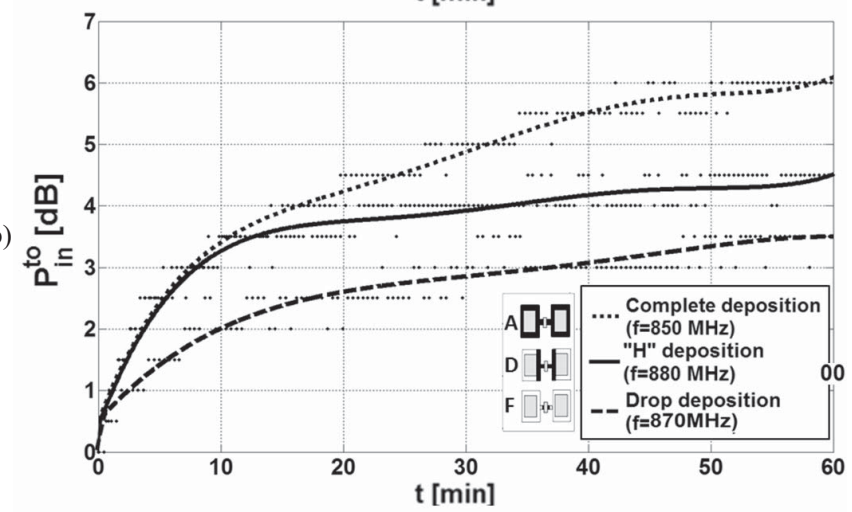

Fig. 10. (a) Measured variation of humidity inside the plastic box. The RH level variation with time has been monitored by means of a digital hygrometer placed inside the box. (b) Measured turn-on power normalized with respect to its initial value, e.g., at ambient $\mathrm{RH}$, for three different polymer depositions into the H-slot. Data (discontinuous dots) have been fitted by a mean square interpolation.

TABLE II

SENSITIVITIES [dB/RH] OF MEASURED TURN-ON AND BACKSCATTER POWER

\begin{tabular}{|c|c||c||c||c|}
\cline { 2 - 5 } \multicolumn{1}{c|}{} & $\mathrm{RH}_{\text {low }}-\mathrm{RH}_{\text {high }}$ & Case $\mathrm{A}$ & Case D & Case $\mathrm{F}$ \\
\hline \multirow{3}{*}{$S_{P_{\text {in }}^{\text {to }}}$} & $50 \%-80 \%$ & 0.13 & 0.12 & 0.08 \\
\cline { 2 - 5 } & $80 \%-100 \%$ & 0.1 & 0.05 & 0.05 \\
\cline { 2 - 5 }$S_{p_{B S}}$ & $50 \%-80 \%$ & 0.18 & 0.2 & 0.12 \\
\cline { 2 - 5 } & $80 \%-100 \%$ & 0.13 & 0.05 & 0.05 \\
\hline
\end{tabular}

responses versus the variation of the humidity. Fig. 11 shows the normalized values of turn-on and backscattered power with respect to the initial condition. The profiles appear almost linear, especially at low humidity grades and so it is possible to extract the sensitivity of the devices, e.g the slope of the linearized curves, as the power difference generated by $1 \%$ change in the RH level

$$
S_{\xi}=\frac{|\Delta \xi|}{|\Delta R H|}=\frac{\left|\xi\left(R H_{\text {high }}\right)-\xi\left(R H_{\text {low }}\right)\right|}{\left|R H_{\text {high }}-R H_{\text {low }}\right|}
$$

where $\xi=\left\{P_{i n}^{t o}, p_{B S}\right\}$. The values for the three depositions of Fig. 11 are listed in Table II. In the lower part of the dynamics range, the backscattered power metric appear more sensitive to humidity variations that the turn-on power indicator, while no remarkable difference may be observed close to the saturation. It is finally worth noticing that the deposition layout corresponding to a single polymer drop (case F) has a nearly halved sensitivity with respect to the full-deposition tag A. However, at least for low humidity grades, its sensitivity is fully comparable with the one achieved in [6], further demonstrating the possibility of reducing the amount of sensor deposition without affecting its performances.

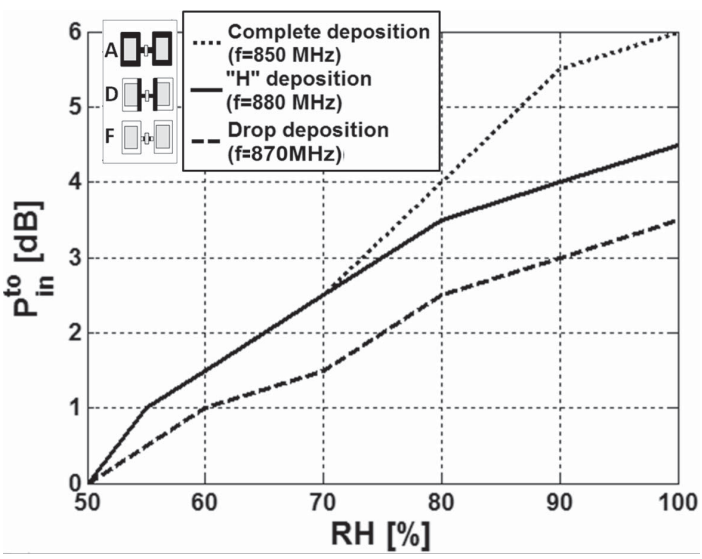

(a)

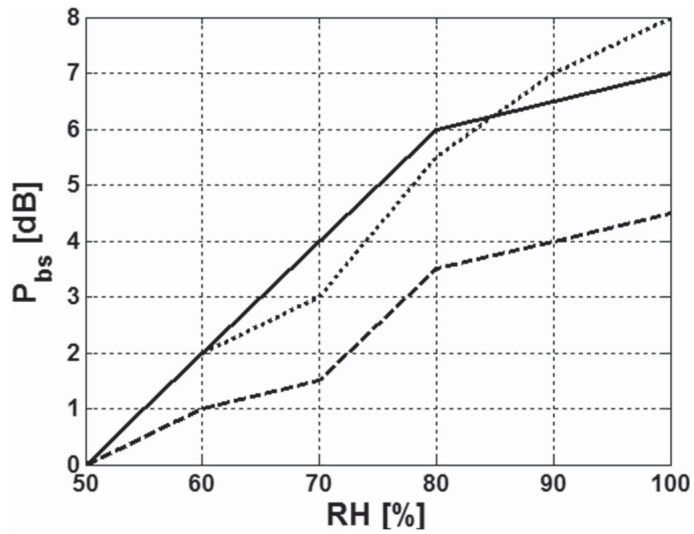

(b)

Fig. 11. Calibration curves of the humidity RFID sensor for some layouts of polymer deposition. (a) Turn-on power. (b) Backscattered power.

\section{RECOVERY AND HYSTERESIS}

Reproducibility, recovery, and hysteresis of the proposed RFID sensor have been tested by set up a cyclic exposure of humidity with two periods of different durations between wet and dry air (Fig. 12). An intermediate tag layout (case D of Fig. 8) has been considered for the purpose of turn-on power and backscattered power measurements. As in the previous case, to improve the readability, a mean-square interpolation is superimposed to the stair-step distribution of the measurement data caused by the coarse turn-on power and backscattered power resolutions of the UHF reader (respectively $0.5 \mathrm{dBm}$ and $0.8 \mathrm{dBm}$ ). The achieved profiles are visible in Fig. 12 for the selected frequency $880 \mathrm{M} \mathrm{Hz}$ which ensures the strongest signals variation during the process. The change of the RFID powers reasonably follows the variation of the humidity. The recovery process at $\mathrm{RH}=50 \%$ takes place after the two cycles of humidity exposure during $T_{1}<t<T_{2}$ and $T_{3}<t<T_{4}$. As the box is removed the relative humidity drops down to the value of ambient air and hence the radio sensor starts its recovery. After $60 \mathrm{~min}$ the recovery can be considered completed. However, just $5 \mathrm{~min}$ are enough for the sensor to recover $3 \mathrm{~dB}$ of turn-on power and $4 \mathrm{~dB}$ of the backscattered power. The effects are reversible, with a negligible hysteresis. Once the baseline value has been reached, the response remains stable with only $1 d B$ difference between the beginning and the end of the process. 
(a)

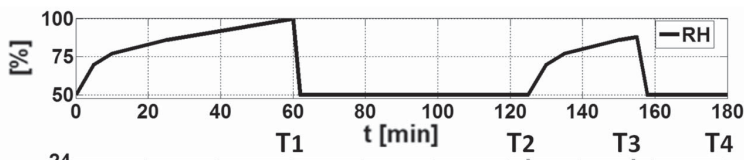

(b)

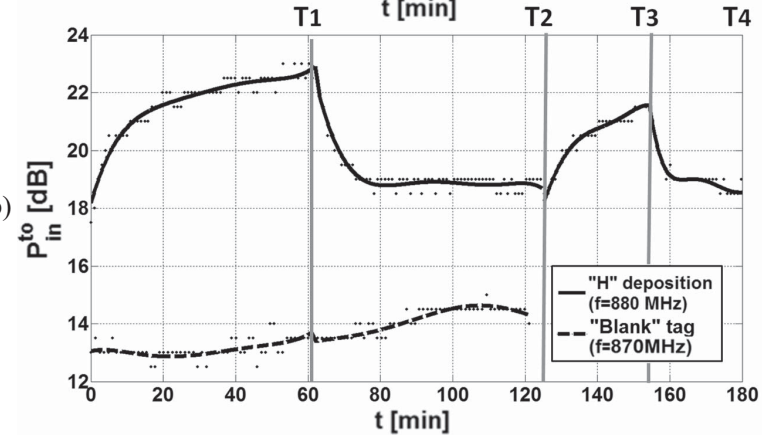

(c)

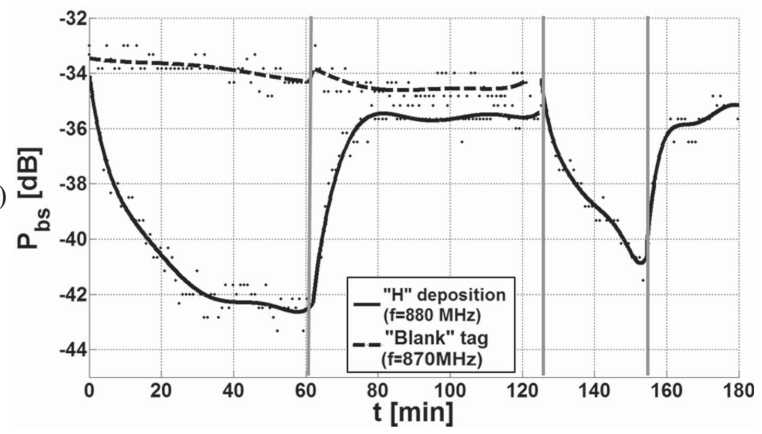

Fig. 12. (a) Measured variation of humidity inside the plastic box. Measured (b) turn-on and (c) normalized backscattered power during cyclic exposures. The power from the "blank" tag is also visible. Here $T_{1}=60 \mathrm{~min}$, $T_{2}=120 \mathrm{~min}, T_{3}=150 \mathrm{~min}$, and $T_{4}=180 \mathrm{~min}$.

Finally, to double-check the true effectiveness of the doped tag against other external bias, not depending on the polymer itself, also a "blank" tag subjected to the same cyclic exposure as before has been considered. The formation of water drops over the copper produces a kind of variation of the response, even in absence of polymer, but the overall measured change, for instance concerning the backscatterd power (see again Fig. 12), is negligible in comparison with the measured dynamics of the true polymer-doped tags.

\section{REAL APPLICATION}

To discuss the response of the proposed humidity sensor in realistic conditions, this has been finally applied to monitor an overnight exposure. The doped tag (case D of Fig. 8) has been placed outside an external wall and interrogated by the RFID reader installed on the inside (Fig. 13) with its antenna attached on the inner side of the windows. The distance between reader and tag is such to enable a robust RFID communication regardless the humidity grade. The $\mathrm{RH}$ and temperature levels have been monitored by means of a digital hygrometer placed outside, in close proximity of the tag. The experiment started at 6 p.m. in the evening on November 21st 2011 in Rome and ended at 2 p.m. in the afternoon on November $22 \mathrm{nd}$. The temperature was relatively stable in a range between $13{ }^{\circ} \mathrm{C}$ and $17{ }^{\circ} \mathrm{C}$.

The results in terms of normalized turn-on and backscattered power are shown in Fig. 14 at $880 \mathrm{MHz}$. The tag's responses

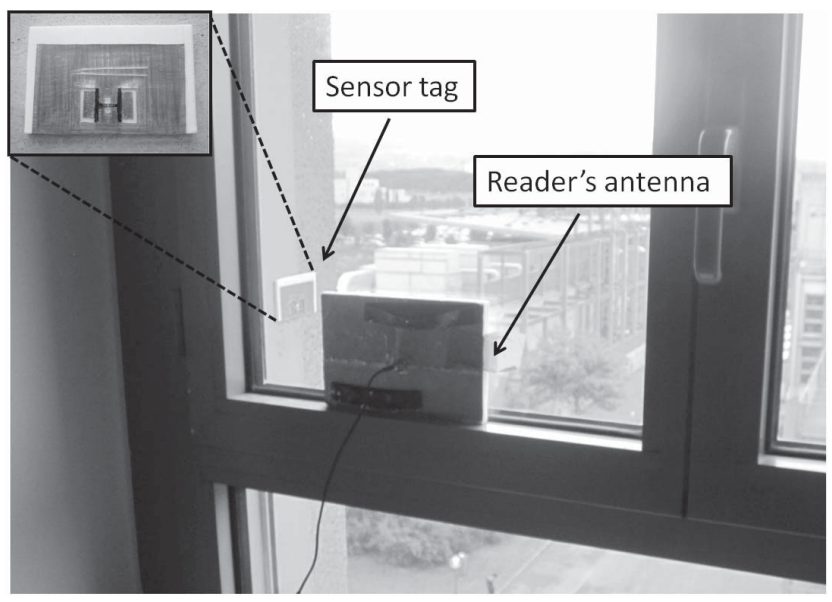

Fig. 13. Measurement setup for the overnight humidity exposure.

(a)

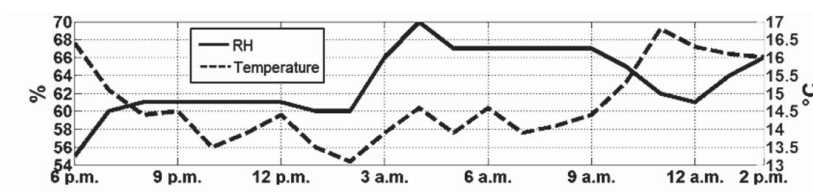

(b)

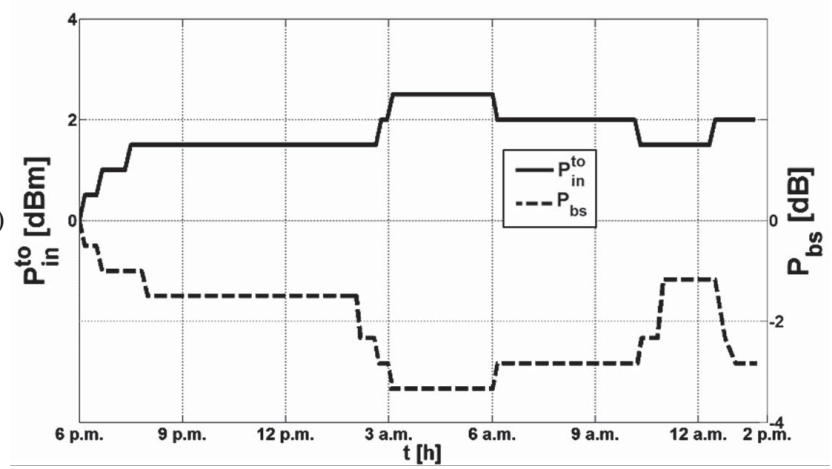

Fig. 14. (a) Measured external temperature and humidity variation during the night. (b) Measured normalized turn-on and backscattered power at $880 \mathrm{MHz}$ during the overnight humidity exposure obtained with a moving window filter interpolation.

reasonably follow the profile of humidity during both increase (night) and reduction (morning). The dynamic range of variation of the sensor is $2.5 \mathrm{~dB}$ for turn-on power and $3 \mathrm{~dB}$ for backscattered power between the minimum (55\%) and the maximum humidity $(70 \%)$ detected during the night. These dynamic ranges are comparable with the previously shown results obtained in the controlled cyclic humidity exposure.

In these measurements cross-sensitivity effects due to atmospheric pressure and temperature have not been investigated. It is known that the d.c. resistivity of PEDOT:PSS is sensitive also to temperature and pressure [10]. However, the effect of these quantities at the investigated frequency is still unknown. On the other hand, air pressure and temperature may be indirectly detected. For instance the dependence of the sensor signal from the temperature is due to several factors including the modulation of the actual water vapor concentration (being the relative humidity dependent on the temperature), the modulation of the water vapour partition coefficient in the polymer matrix, and finally, the intrinsic sensitivity of the material to the temperature. 


\section{CONCLUSION}

The complete integration of PEDOT:PSS into RFID tag has been here reported for the first time aiming to design and test a passive radio-sensor suitable to monitor the local humidity. Communication, sensing performances and cyclic exposures have been jointly analyzed, leading to a fully working prototype, whose sensitivity and dynamic range can be controlled by the amount and the displacement of polymer into the sensing slots. In particular, laboratory experiments demonstrate that just a single polymer drop, deposited right behind the microchip, is able to provide more than $3 \mathrm{~dB}$ dynamic range and up to $0.12 \mathrm{~dB} / \mathrm{RH}$ sensitivity at the price of a nearly negligible worsening of communication range (less than $20 \%$ ).

A commercial polymer formulation has been used, but for future research the polymer can be chemically modified with appropriate functional groups in order to increase the sensitivity (and then the resolution), the selectivity and the response/recovery time. Also different Pedot:PSS ratio should be analyzed for the optimization of the sensor.

Figures 12 and 14 show that the absorption of water vapour is completely reversible. So the exposure to changes of relative humidity is not expected to age the sensor. Instead, an important issue to be better investigated in future research is the adhesion of the polymer layer onto the substrate, which could have a significant impact for the sensor's lifetime. A proper investigation of the cross-sensitivity, for instance to other polar volatile compounds, will be a necessary next step of this research.

The proposed sensor layout is completely unspecific; the PEDOT:PSS itself, being sensitive to several parameters (temperature, humidity, organic gases) could be used for different sensing purposes or the tag could be doped by means of different sensitive chemical species eventually properly functionalized.

Thanks to the presence of the ground plane, which decouples the antenna from its location, this kind of tag is moreover suitable to environmental and body-centric applications. It could be disseminated into the environment, e.g integrated into walls, infrastructures and devices or attached onto the human body as well as embedded into plasters or bandages to remotely monitor the healing grade of wounds.

Beside the promising results, this class of chemical-doped RFID tags is however still prone to considerable improvements concerning the technology process to deposit the chemical load and not least, concerning the interrogation modalities and instruments. Depending on the preparation technique and process, the properties and the behavior of the sensors can indeed vary significantly, and this is a very crucial issue for the large-scale production of chemical-based devices. Regarding the sensing detection, instead, the bottleneck of this platform is the resolution and stability of the RFID reader. Actual commercial UHF readers are designed to item labeling and not to sensing, and therefore significantly better performances are expected with dedicated receivers having a finer resolution in the power tuning and in the analog to digital converter.

\section{REFERENCES}

[1] G. Marrocco, "Pervasive electromagnetics: Sensing paradigms by passive RFID technology," IEEE Wireless Commun., vol. 17, no. 6, pp. 10-17, Dec. 2010.

[2] C. Occhiuzzi, A. Rida, G. Marrocco, and M. Tentzeris, "RFID passive gas sensor integrating carbon nanotubes," IEEE Trans. Microw. Theory Tech., vol. 59, no. 10, pp. 2674-2684, Oct. 2011.

[3] S. Caizzone, C. Occhiuzzi, and G. Marrocco, "Multi-chip RFID antenna integrating shape-memory alloys for detection of thermal thresholds," IEEE Trans. Antennas Propag., vol. 59, no. 7, pp. 2488-2494, Jul. 2011.

[4] B.-S. Atiyeh and S.-N. Hayek, "Moisture and wound healing," J. Plaies Cicatrisat., vol. 46, pp. 7-11, Dec. 2004.

[5] J. Siden, J. Gao, and B. Neubauer, "Microstrip antennas for remote moisture sensing using passive RFID," in Proc. Microw. Conf., Dec. 2009, pp. 2375-2378.

[6] J. Virtanen, L. Ukkonen, T. Bjorninen, A. Elsherbeni, and L. Sydandnheimo, "Inkjet-printed humidity sensor for passive UHF RFID systems," IEEE Trans. Instrum. Meas., vol. 60, no. 8, pp. 2768-2777, Aug. 2011.

[7] H. Bai and G. Shi, "Gas sensors based on conducting polymers," Sensors, vol. 7, no. 3, pp. 267-307, 2007.

[8] Y. Sakai, Y. Sadaoka, and M. Matsuguchi, "Humidity sensors based on polymer thin films," Sensors Actuat. B, Chem., vol. 35, nos. 1-3, pp. 85-90, 1996.

[9] A. Elschner, S. Kirchmeyer, W. Lovenich, U. Merker, and K. Reuter, PEDOT: Principles and Applications of an Intrinsically Conductive Polymer. Boca Raton, FL: CRC Press, 2010.

[10] T. Kinkeldei, C. Zysset, K. Cherenack, and G. Troster, "A textile integrated sensor system for monitoring humidity and temperature," in Proc. 16th Int. Solid-State Sensors, Actuat. Microsyst. Conf., Jun. 2011, pp. 1156-1159.

[11] J. Reboun, A. Hamacek, T. Dzugan, and M. Kroupa, "Sensorial characteristics of conductive polymers," in Proc. 32nd Spring Seminor Int. Electron. Technol., May 2009, pp. 1-5.

[12] C. Occhiuzzi, S. Cippitelli, and G. Marrocco, "Modeling, design and experimentation of wearable RFID sensor tag," IEEE Trans. Antennas Propag., vol. 58, no. 8, pp. 2490-2498, Aug. 2010.

[13] Clevios ph500. (2010) [Online]. Available: http://clevios.com/

[14] M. J. J. Kamil, "Flexible temperature sensor integrated with RFID tag," in Proc. 13th Int. Ph.D. Workshop OWD, Oct. 2011.

[15] N. Kirsch, N. Vacirca, T. Kurzweg, A. Fontecchio, and K. Dandekar, "Performance of transparent conductive polymer antennas in a MIMO ad-hoc network," in Proc. 6th Int. Conf. Wireless Mobile Comput., Netw. Commun., Oct. 2010, pp. 9-14.

[16] N. Kirsch, N. Vacirca, E. Plowman, T. Kurzweg, A. Fontecchio, and K. Dandekar, "Optically transparent conductive polymer RFID meandering dipole antenna," in Proc. IEEE Int. Conf. RFID, Apr. 2009, pp. 278-282.

[17] G. Marrocco and F. Amato, "Self-sensing passive RFID: From theory to tag design and experimentation," in Proc. Eur. Microw. Conf., Oct. 2009, pp. 1-4.

Sabina Manzari, photograph and biography are not available at the time of publication.

Cecilia Occhiuzzi, photograph and biography are not available at the time of publication.

Shankar Nawale, photograph and biography are not available at the time of publication.

Alexandro Catini, photograph and biography are not available at the time of publication.

Corrado Di Natale, photograph and biography are not available at the time of publication.

Gaetano Marrocco, photograph and biography are not available at the time of publication. 